\title{
Robust and Simple Authentication Protocol for Secure Communication on the Web
}

\author{
Eun-Jun Yoon ${ }^{1}$, Woo-Hun $\mathrm{Kim}^{2}$, and Kee-Young Yoo ${ }^{1, \star}$ \\ 1 Department of Computer Engineering, Kyungpook National University, \\ Daegu 702-701, Republic of Korea \\ ejyoon@infosec.knu.ac.kr, yook@knu.ac.kr \\ 2 Department of Information Security, Kyungpook National University, \\ Daegu 702-701, Republic of Korea \\ whkim@infosec.knu.ac.kr
}

\begin{abstract}
User authentication is an important part of security, along with confidentiality and integrity, for systems that allow remote access over untrustworthy networks, such as the Internet Web environment. In 2005, Chien-Wang-Yang (CWY) pointed out that Chien-Jan's ROSI protocol required state synchronization between the client and the server, and then its state-synchronization property was vulnerable to the Denial of Service (DoS) attack. Furthermore, they proposed an improved protocol that conquered the weaknesses and extended its key agreement functions, and improved the server's performance. Nevertheless, CWY's improved ROSI protocol does not provide perfect forward secrecy and is vulnerable to a Denning-Sacco attack. Accordingly, the current paper demonstrates that CWY's protocol does not provide perfect forward secrecy and is susceptible to a Denning-Sacco attack. We then present an enhanced protocol to isolate such problems.
\end{abstract}

Keyword: Cryptography, Security, Authentication, Smart card, Key establishment, Forward Secrecy, Denning-Sacco attack

\section{Introduction}

Perhaps the environment in which authentication is the most crucial is the Internet Web environment. Therefore, user authentication is an important part of security, along with confidentiality and integrity, for systems that allow remote access over untrustworthy networks, such as the Internet Web environment. Every day, there are vast numbers of authentication processes taking place. From logging in to a corporate system or database, reading your e-mail, making a purchase online, or performing a financial transaction, each of these common tasks require an authentication phase. As such, a remote password authentication scheme authenticates the legitimacy of users over an insecure channel, where the password is often regarded as a secret shared between the remote system and the user. Based on knowledge of the password, the user can create and send a

\footnotetext{
* Corresponding author. Tel.: +82-53-950-5553; Fax: +82-53-957-4846
} 
valid login message to a remote system in order to gain access. Meanwhile, the remote system also uses the shared password to check the validity of the login message and authenticates the user. However, a password-based protocol is vulnerable to a password guessing attack, replay attack, stolen-verifier problem, denial of service (DoS) attack or a forgery attack [1][2]. These attacks affect the integrity of the SAS protocol [3], the revised SAS-1 [4], the revised SAS-2 [4] or the OSPA protocol [5].

ISO 10202 standards have been established for the security of financial transaction systems using integrated circuit cards (IC cards or smart cards). A smart card originates from an IC memory card used which has been in the industry for about 10 years [6][7]. The main characteristics of a smart card are its small size and low-power consumption. Generally speaking, a smart card contains a microprocessor which can quickly manipulate logical and mathematical operations, RAM which is used as a data or instruction buffer, and ROM which stores the user's secret key and the necessary public parameters and algorithmic descriptions of the executing programs. The merits of a smart card for password authentication are its high simplicity and its efficiency regarding of the log-in and authentication processes.

Based upon low-cost smart cards that support only simple hashing operations, ROSI [8] is a highly efficient password-based authentication protocol. Its simplicity, resistance to existing known attacks and high performance make it much more attractive than its counterparts. In 2005, Chien-Wang-Yang [9], however, pointed out that the ROSI protocol requires state synchronization between the client and the server, and then its state-synchronization property makes it vulnerable to a Denial of Service (DoS) attack. In addition, they proposed an improved protocol that overcomes its weaknesses, extends its key agreement functions, and improves the server's overall performance.

Nevertheless, CWY's improved ROSI protocol does not provide perfect forward secrecy [10] and is vulnerable to a Denning-Sacco attack [11]. Accordingly, the current paper demonstrates that CWY's protocol does not provide perfect forward secrecy and susceptible to a Denning-Sacco attack, where an attacker can easily obtain legal client's secret value. We then present an enhanced protocol to isolate such problems.

The remainder of the paper is organized as follows: Section 2 defines the security properties. Section 3 briefly reviews CWY's improved ROSI protocol, then Section 4 demonstrates the security weaknesses of CWY's protocol. The proposed ROSI protocol is presented in Section 5, while Section 6 discusses the security and efficiency of the proposed protocol. The conclusion is given in Section 7 .

\section{Security Properties}

The following security properties of the authentication protocols should be considered [1]. 
(1) Guessing attack: A guessing attack involves an adversary (randomly or systematically) trying long-term private keys (e.g. user password or server secret key), one at a time, in the hope of finding the correct private key. Ensuring long-term private keys chosen from a sufficiently large space can reduce exhaustive searches. Most users, however, select passwords from a small subset of the full password space. Such weak passwords with low entropy are easily guessed by using the so-called dictionary attack.

(2) Replay attack: A replay attack is an offensive action in which an adversary impersonates or deceives another legitimate participant through the reuse of information obtained in a protocol.

(3) Stolen-verifier attack: In most applications, the server stores verifiers of users' passwords (e.g. hashed passwords) instead of the clear text of passwords. The stolen-verifier attack means that an adversary who steals the password-verifier from the server can use it directly to masquerade as a legitimate user in a user authentication execution.

In addition, the following security properties of session key agreement protocols should be considered since they are often desirable in some environments [1].

(1) Denning-Sacco attack: The Dennig-Sacco attack is where an attacker compromises an old session key and tries to find a long-term private key (e.g. user password or server private key) or other session keys.

(2) Implicit key authentication: Implicit key authentication is the property obtained when identifying a party based on a shared session key, which assures that no other entity than the specifically identified entity can gain access to the session key.

(3) Explicit key authentication: Explicit key authentication is the property obtained when both implicit key authentication and key confirmation hold.

(4) Mutual authentication: Mutual authentication means that both the client and server are authenticated to each other within the same protocol.

(5) Perfect forward secrecy: Perfect forward secrecy means that if a longterm private key (e.g. user password or server private key) is compromised, this does not compromise any earlier session keys.

\section{Review of CWY's ROSI Protocol}

This section briefly reviews CWY's ROSI protocol. Some of the notations used in this paper are defined as follows:

- ID: public user identity of client.

- $P W$ : secret and possibly weak user password.

$-x$ : server's strong secret key.

- $N_{c}, N_{s}$ : random nonce chosen by the client and server, respectively.

- $p, q$ : large prime numbers $p$ and $q$ such that $q \mid p-1$. 
- $g$ : generator with order $q$ in the Galois Field $G F(p)$, in which the DiffieHellman problem is considered hard.

- $c, s$ : session-independent random exponents $\in[1, q-1]$ chosen by the client and server, respectively.

- $K$ : shared fresh session key computed by the client and server.

- $h(\cdot)$ : secure one-way hash function.

- $\|$ : concatenation operation.

- $\oplus$ : bit-wise XOR operation.

CWY's ROSI protocol consists of two phases; the registration phase, and the authentication and key establishment phase.

\subsection{Registration Phase}

(1) The user submits his $I D$ and $P W$ to the server through a secure channel.

(2) The server issues the user a smart card that stores $R=h(x \| I D) \oplus P W$ and $h(\cdot)$.

\subsection{Authentication and Key Establishment Phase}

The user who has obtained the server-issued smart card can perform the following steps to log on to the server and establish a fresh session key with the server. CWY's authentication and key establishment phase is illustrated in Figure 1.

(1) Client $\rightarrow$ Server: $I D, c_{1}, c_{2}$

The user inserts his smart card into the terminal and inputs his $I D$ and $P W$. The card then extracts $h(x \| I D)$ by computing $R \oplus P W$, chooses a random nonce $N_{c}$ and computes $c_{1}=h(x \| I D) \oplus N_{c}$ and $c_{2}=h\left(I D \| N_{c}\right)$. The card sends $\left(I D, c_{1}, c_{2}\right)$ to the server.

(2) Server $\rightarrow$ Client: $c_{3}, c_{4}$

Based on the received $I D$, the server computes $h(x \| I D)$, extracts the nonce $N_{c}$ by computing $c_{1} \oplus h(x \| I D)$ and verifies the data by checking whether $h\left(I D \| N_{c}\right)$ equals $c_{2}$. If the verification fails, then the server stops the authentication; otherwise, it chooses a random nonce $N_{s}$ and computes the session key $K=h\left(h(x \| I D)\left\|N_{c}\right\| N_{s}\right), c_{3}=h\left(N_{c}\right) \oplus N_{s}$ and $c_{4}=h\left(K\left\|N_{c}\right\| N_{s}\right)$. The server finally sends $\left(c_{3}, c_{4}\right)$ to the client.

(3) Client $\rightarrow$ Server: $c_{5}$

Upon receiving $\left(c_{3}, c_{4}\right)$, the client first derives $N_{s}=c_{3} \oplus h\left(N_{c}\right)$, computes the session key $K=h\left(h(x \| I D)\left\|N_{c}\right\| N_{s}\right)$ and then verifies whether $h\left(K\left\|N_{c}\right\| N_{s}\right)$ equals the received $c_{4}$. If the verification fails, it stops the authentication; otherwise, it sends back $c_{5}=h\left(N_{s}\left\|N_{c}\right\| K\right)$ to the server.

(4) Upon receiving the data $c_{5}$, the server verifies whether $c_{5}$ equals $h\left(N_{s}\left\|N_{c}\right\| K\right)$. If the verification fails, it refuses the request; otherwise, mutual authentication is completed and the fresh session key $K$ is confirmed. 
Shared information: Hash function $h(\cdot)$.

Information held by Client: Identity $I D$. Password $P W$. Smart card.

Information held by Server: Secret key $x$.

\section{Client}

\section{Server}

Input $I D, P W$

Extract $h(x \| I D)=R \oplus P W$

Choose $N_{c}$

Compute $c_{1}=h(x|| I D) \oplus N_{c}$

Compute $c_{2}=h\left(I D \| N_{c}\right)$

$\stackrel{I D, c_{1}, c_{2}}{\longrightarrow}$

Compute $h(x \| I D)$

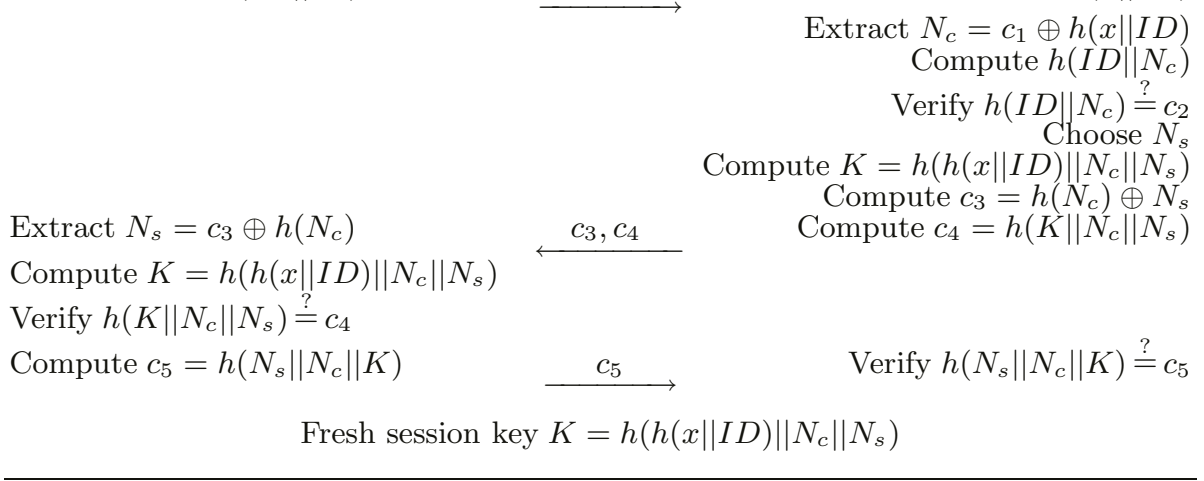

Fig. 1. CWY's authentication and key establishment phase

\section{Cryptanalysis of CWY's ROSI Protocol}

This section shows that CWY's improved ROSI protocol does not provide perfect forward secrecy and is vulnerable to a Denning-Sacco attack.

\subsection{Perfect Forward Secrecy Problem}

Perfect forward secrecy is a very important security requirement in evaluating a strong protocol. A protocol with perfect forward secrecy assures that even if one entity's long-term key is compromised, it will never reveal any session keys used before. For example, the well-known Diffie-Hellman key agreement scheme [3] can provide perfect forward secrecy. CWY's ROSI protocol, however, does not provide it because once the secret key $x$ of the server is disclosed, all previous fresh session keys $K$ will also be opened and hence previous communication messages will be learned.

In the CWY's ROSI protocol, suppose an attacker $E$ obtains the secret key $x$ from the compromised server and intercepts transmitted values $\left(I D, c_{1}, c_{2}\right.$, $\left.c_{3}\right)$, then $E$ can compute $h(x \| I D)$ and extract the client's random nonce $N_{c}$ by computing $c_{1} \oplus h(x \| I D)$. Using the extracted $N_{c}, E$ can also extract server's random nonce $N_{s}$ by computing $c_{3} \oplus h\left(N_{c}\right)$. Finally, $E$ can compute the session key $K=h\left(h(x \| I D)\left\|N_{c}\right\| N_{s}\right)$ by using $h(x \| I D), N_{c}$ and $N_{s}$. Obviously, CWY's ROSI protocol does not provide perfect forward secrecy. 


\subsection{Denning-Sacco Attack}

This attack arises from the fact that the compromise of a random nonce or fresh session key enables the protocol to be compromised. Such attacks have long been known. Please refer the Denning-Sacco attack in [1]. If $N_{c}$ becomes known to an attacker $E$, then $E$ can use this knowledge to impersonate the client to the server in this instance of the protocol. This is a typical and uncontroversial assumption for an authentication protocol; however, we show below that if such a random nonce $N_{c}$ is ever disclosed, even long after the protocol is executed, then a serious attack is possible.

In the CWY's ROSI protocol, suppose $E$ has intercepted messages $c_{1}$ of the protocol and learned the random nonce $N_{c}$ in $c_{1}$ by some means. Then, knowledge of $N_{c}$ will enable $h(x \| I D)$ to be discovered from $c_{1}$ by computing $c_{1} \oplus N_{c}$ (since $N_{c}$ included in $c_{1}$ is known to $E$ ). Compromise of the user's secret value $h(x \| I D)$ will enable the attacker to impersonate the client freely. For example, $E$ chooses a random nonce $N_{e}$ and computes $c_{1}^{*}=h(x|| I D) \oplus N_{e}$ and $c_{2}^{*}=h\left(I D \| N_{e}\right)$, by using the compromised user's secret value $h(x \| I D)$. If $E$ sends these modified values $\left(I D, c_{1}^{*}, c_{2}^{*}\right)$ to the server, then the server will authenticate $E$ by performing the authentication and key establishment phase. Therefore, CWY's ROSI protocol is obviously insecure against a Denning-Sacco attack.

\section{Proposed ROSI Protocol}

This section proposes an improved ROSI protocol by providing perfect forward secrecy in order to overcome the above mentioned problems with CWY's protocol. The improved protocol also consists of two phases; the registration phase, and the authentication and key establishment phase.

\subsection{Registration Phase}

(1) The user submits his $I D$ and $P W$ to the server through a secure channel.

(2) The server issues the user a smart card that stores $R=h(x \| I D) \oplus P W$, $h(\cdot), p$ and $g$.

\subsection{Authentication and Key Establishment Phase}

The user who has obtained the server-issued smart card can perform the following steps to log on to the server and establish fresh session keys with the server. The proposed authentication and key establishment phase is illustrated in Figure 2.

(1) Client $\rightarrow$ Server: $I D, c_{1}, c_{2}$

The user inserts his smart card into the terminal and inputs his $I D$ and $P W$. The card then extracts $h(x \| I D)$ by computing $R \oplus P W$, chooses a random exponent $c \in Z_{p}^{*}$ and computes $c_{1}=g^{c}(\bmod p)$ and $c_{2}=h\left(h(x \| I D) \| g^{c}\right)$. The card sends $\left(I D, c_{1}, c_{2}\right)$ to the server. 
Shared information: Generator $g$ of $Z_{p}^{*}$ of prime order $q$. Hash function $h(\cdot)$. Information held by Client: Identity $I D$. Password $P W$. Smart card.

Information held by Server: Secret key $x$.

\section{Client}

\section{Server}

Input $I D, P W$

Extract $h(x \| I D)=R \oplus P W$

Choose $c \in Z_{p}^{*}$

Compute $c_{1} \stackrel{p}{=} g^{c}(\bmod p)$

Compute $c_{2}=h\left(h(x \| I D) \| g^{c}\right)$

$\stackrel{I D, c_{1}, c_{2}}{\longrightarrow}$

Compute $h(x|| I D)$

Verify $h\left(h(x \| I D) \| c_{1}\right) \stackrel{?}{=} c_{2}$

Choose $s \in Z_{p}^{*}$

Compute $K=g^{c s}(\bmod p)$

Compute $c_{3}=g^{s}(\bmod p)$

Compute $K=\left(c_{3}\right)^{c}=g^{c s}(\bmod p)$

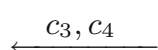

Compute $c_{4}=h\left(K \| c_{1}\right)$

Verify $h\left(K \| g^{c}\right) \stackrel{?}{=} c_{4}$

Compute $c_{5}=h\left(K \| c_{3}\right)$

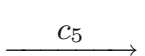

Verify $h\left(K \| g^{s}\right) \stackrel{?}{=} c_{5}$

Fresh session key $K=g^{c s}(\bmod p)$

Fig. 2. Proposed authentication and key establishment phase

(2) Server $\rightarrow$ Client: $c_{3}, c_{4}$

Based on the received $I D$, the server computes $h(x \| I D)$ and verifies the data by checking whether $h\left(h(x \| I D) \| c_{1}\right)$ equals $c_{2}$. If the verification fails, then the server stops the authentication; otherwise, it chooses a random exponent $s \in Z_{p}^{*}$ and computes the session key $K=g^{c s}(\bmod p), c_{3}=g^{s}(\bmod p)$ and $c_{4}=h\left(K \| c_{1}\right)$. The server finally sends $\left(c_{3}, c_{4}\right)$ to the client.

(3) Client $\rightarrow$ Server: $c_{5}$

Upon receiving $\left(c_{3}, c_{4}\right)$, the client first computes the session key $K=\left(c_{3}\right)^{c}=$ $g^{c s}(\bmod p)$ and then verifies whether $h\left(K \| g^{c}\right)$ equals the received $c_{4}$. If the verification fails, it stops the authentication; otherwise, it sends back $c_{5}=$ $h\left(K \| c_{3}\right)$ to the server.

(4) Upon receiving the data $c_{5}$, the server verifies whether $c_{5}$ equals $h\left(K \| g^{s}\right)$. If the verification fails, it refuses the request; otherwise, mutual authentication is completed and the fresh session key $K$ is confirmed.

\section{Security Analysis and a Comparison of Performance}

This section discusses the security and efficiency of the proposed ROSI protocol.

\subsection{Security Analysis}

This subsection analyzes the security of the proposed ROSI protocol. At first, we define the security terms needed for the analysis of the proposed ROSI protocol. 
Definition 1. A weak secret (password) is a value of low entropy $W(k)$, which can be guessed in polynomial time.

Definition 2. A strong secret key is a value of high entropy $H(k)$, which cannot be guessed in polynomial time.

Definition 3. A secure one-way hash function $y=h(x)$ is one where given $x$ it is easy to compute $y$ and where given $y$ it is hard to compute $x$.

Definition 4. The discrete logarithm problem (DLP) is explained by the following: Given a prime $p$, a generator $g$ of $Z_{p}^{*}$, and an element $\beta \in Z_{p}^{*}$, find the integer $\alpha, 0 \leq \alpha \leq p-2$, such that $g^{\alpha} \equiv \beta(\bmod p)$.

Definition 5. The Diffie-Hellman problem (DHP) is explained by the following: Given a prime $p$, a generator $g$ of $Z_{p}^{*}$, and elements $g^{c}(\bmod p)$ and $g^{s}(\bmod p)$, find $g^{c s}(\bmod p)$.

Here, six security properties: Guessing attack, replay attack, stolen-verifier attack, Denning-Sacco attack, mutual authentication, and perfect forward secrecy, would be considered for the proposed ROSI protocol. Under the above definitions, the following theorems are used to analyze the six security properties in the proposed protocol.

Theorem 1. The proposed ROSI protocol can resist the server's secret key guessing attack.

Proof. An attacker $E$ can intercept a login request message $\left(I D, c_{1}, c_{2}\right)$ sent by the client in Step (1) over a public network. Due to Definition 3, however, that a secure one-way hash function is computationally difficult to invert, he cannot derive the client's secret value $h(x \| I D)$ from $c_{2}$. Suppose that an attacker obtains the client's secret value $h(x \| I D)$. Due to Definitions 2 and 3, however, it is also extremely hard for any attacker to derive the server's strong secret key $x$ from $h(x \| I D)$.

Theorem 2. The proposed ROSI protocol can resist the replay and an impersonation attack.

Proof. An attacker $E$ can intercept $\left(I D, c_{1}, c_{2}\right)$ and use it to impersonate the client when sending the next login message. For a random challenge, however, the $g^{c}$ and $g^{s}$ separately generated by the client and server are different every time. Since the client and server always verify the integrity of the fresh session key $K$ by checking $c_{4}$ and $c_{5}$, the replayed messages can be detected by the client and server, respectively. Furthermore, obtaining random exponents $c$ and $s$ from $g^{c}$ and $g^{s}$ is computationally infeasible, as it is a discrete logarithm problem of Definition 4. Therefore, $E$ cannot compute $K$ without knowing $c$ and $s$. In addition, it is also computationally infeasible to obtain the fresh session key $K=g^{c s}$ from $g^{c}$ and $g^{s}$, as it is the Diffie-Hellman problem of Definition 5. As a result, $E$ cannot impersonate the client or the server. 
Theorem 3. The proposed ROSI protocol can resist the stolen-verifier attack.

Proof. Servers are always the target of attacks. An attacker may try to steal or modify the verification table stored in the server. If the verification table is stolen by an attacker, the attacker may masquerade as a legitimate user. If the verification table is modified, a legitimate user cannot successfully login to the system. This results in a denial-of-service attack. The proposed protocol is a nonce-based authentication protocol, but it does not require a verification table in the server. Obviously, the proposed protocol can prevent the stolen-verifier attack.

Theorem 4. The proposed ROSI protocol can resist the Denning-Sacco attack.

Proof. Although an attacker $E$ obtains $g^{c}, g^{s}$ and fresh session key $g^{c s}, E$ cannot obtains the client's secret value $h(x \| I D)$ from $c_{2}=h\left(h(x \| I D) \| g^{c}\right)$ because $x$ is a strong secret key by Definition 1 and $h(\cdot)$ is a secure one-way hash function by Definition 3. Obviously, the proposed protocol can prevent the Denning-Sacco attack.

Theorem 5. The proposed ROSI protocol provides mutual authentication.

Proof. Mutual authentication means that both the client and server are authenticated to each other within the same protocol, while explicit key authentication is the property obtained when both implicit key authentication and key confirmation hold. As such, the improved protocol uses the Diffie-Hellman key exchange algorithm to provide mutual authentication, then the key is explicitly authenticated by a mutual confirmation fresh session key $K$.

Theorem 6. The proposed ROSI protocol provides perfect forward secrecy.

Proof. Even if both client's password PW and server's secret key $x$ are compromised simultaneously, an attacker $E$ can derive only the fresh session key at this time. Previous fresh session keys cannot be opened because the fresh session key is constructed under the Diffie-Hellman key agreement scheme. That is, it is computationally infeasible to obtain the fresh session key $K=g^{c s}$ from $g^{c}$ and $g^{s}$ by Definition 5 . So the proposed ROSI protocol provides perfect forward secrecy.

The security properties of CWY's ROSI protocol, and the proposed ROSI protocol are summarized in Table 1.

\subsection{Performance Comparison}

The computational costs of CWY's ROSI protocol and the proposed ROSI protocol in the authentication and key establishment phase are summarized in Table 2. The computational costs of the registration phase are the same. In the authentication and key establishment phase, CWY's protocol requires a total of 
Table 1. Comparisons of security properties

\begin{tabular}{|l|c|c|}
\hline & CWY's protocol & Proposed protocol \\
\hline \hline Guessing attack & Secure & Secure \\
\hline Replay attack & Secure & Secure \\
\hline Impersonation attack & Secure & Secure \\
\hline Stolen-verifier attack & Secure & Secure \\
\hline Denial of Service attack & Secure & Secure \\
\hline Denning-Sacco attack & Insecure & Secure \\
\hline Mutual authentication & Provided & Provided \\
\hline Perfect forward secrecy & No provided & Provided \\
\hline
\end{tabular}

Table 2. Comparisons of computational costs

\begin{tabular}{|l|c|c|c|c|}
\hline \multirow{2}{*}{} & \multicolumn{2}{|c|}{ CWY's protocol } & \multicolumn{2}{c|}{ Proposed protocol } \\
\cline { 2 - 5 } & Client & Server & Client & Server \\
\hline \hline Exponent operation & 0 & 0 & 2 & 2 \\
\hline Hash operation & 4 & 6 & 3 & 4 \\
\hline XOR operation & 3 & 3 & 1 & 0 \\
\hline
\end{tabular}

ten hashing operations and six exclusive-or operations, but the proposed protocol requires a total of two exponent operations, seven hashing operations and one exclusive-or operations. Two exponent operations are needed to prevent a Denning-Sacco attack and to provide perfect forward secrecy.

When considering hashing and exclusive-or operations, in CWY's protocol, one hashing and one exclusive-or operation for the server are required for a user to register and get his smart card. In the authentication and key establishment phase, four hashing and three exclusive-or operations for the client and six hashing and three exclusive-or operations for the server are required. In the proposed protocol, however, one hashing and one exclusive-or operations in the server are required for a user to register and get his smart card. In the authentication and key establishment phase, three hashing and one exclusive-or operations in the client and four hashing operations in the server are required. Obviously, the proposed protocol is more efficient than CWY's protocol.

\section{Conclusions}

The current paper demonstrated that CWY's improved ROSI protocol does not provide perfect forward secrecy and is susceptible to a Denning-Sacco attack. We then presented an enhancement to the protocol in order to isolate such problems. The proposed ROSI protocol can resist various attacks including guessing, 
replay, stolen-verifier, and Denning-Sacco. In addition mutual authentication and perfect forward secrecy are provided. The computational costs also are significantly less than those of the CWY's protocol. As a result, in contrast to CWY's protocol and the existing SAS-like authentication protocols [3-5, 8], the proposed ROSI protocol can efficiently and securely perform key agreement for secure communication on the Internet Web environment.

\section{Acknowledgements}

This research was supported by the MIC (Ministry of Information and Communication), Korea, under the ITRC (Information Technology Research Center) support program supervised by the IITA (Institute of Information Technology Assessment).

\section{References}

1. Menezes, A.J., Oorschot, P.C., Vanstone, S.A.: Handbook of Applied Cryptograph. CRC Press. New York (1997)

2. Lin, C.L., Hwang, T.: A Password Authentication Scheme with Secure Password Updation. Computers \& Security. Vol. 22. No. 1. (2003) 68-72

3. Sandirigama, M., Shimizu, A., Noda, M.T.: Simple and Secure Password Authentication Protocol (SAS). IEICE Transactions on Communications. Vol. E83-B. No. 6. (2000) 1363-1365

4. Kamioka, T., Shimizum, A.: The Examination of the Security of SAS One-time Password Authentication. IEICE Technical Report. OFS2001-48. No. 435. (2001) 53-58

5. Lin, C.L., Sun, H.M., Hwang, T.: Attacks and Solutions on Strong-password Authentication. IEICE Transactions on Communications. Vol. E84-B. No. 9. (2001) 2622-2627

6. Peyret, P., Lisimaque, G., Chua, T.Y.: Smart Cards Provide Very High Security and Flexibility in Subscribers Management. IEEE Transactions on Consumer Electronics. Vol. 36. No. 3. (1990) 744-752

7. Sternglass, D.: The Future Is in the PC Cards. IEEE Spectrum. Vol. 29. No. 6. (1992) 46-50

8. Chien, H.Y., Jan, J.K.: Robust and Simple Authentication Protocol. The Computer Journal. Vol. 46. No. 2. (2003) 193-201

9. Chien, H.Y., Wang, R.C., Yang, C.C.: Note on Robust and Simple Authentication Protocol. The Computer Journal. Vol. 48. No. 1. (2005) 27-29

10. Steiner, M., Tsudik, G., Waidner, M.: Refinement and Extension of Encrypted Key Exchange. ACM Operating Systems Review. Vol. 29. No. 3. (1995) 22-30

11. Denning, D., Sacco, G.: Timestamps in Key Distribution Systems. Communications of the ACM. Vol. 24. (1981) 533-536 